\title{
The design of an atmospheric pressure ionization/time-of-flight mass spectrometer using a beam deflection method
}

\author{
Ce $\mathrm{Ma}^{\mathrm{a}}{ }^{\mathrm{a}}$ Steven M. Michael, Mingta Chien, Jianzhong Zhu, \\ and David M. Lubman \\ Department of Chemistry, University of Michigan, Ann Arbor, Michigan 48109
}

(Received 3 June 1991; accepted for publication 2 October 1991)

\begin{abstract}
A novel time-of-flight (TOF) mass spectrometer configuration has been designed which can be interfaced to a continuous ion beam source produced by atmospheric pressure ionization. The TOF device uses a beam deflection method to sweep the ion beam past a slit placed near the ionization source in order to generate a start pulse for TOF detection.

The beam sweep technique is modeled by computer simulation and optimized for the various experimental parameters. Nonvolatile samples are injected into the TOF device using liquid injection into a glow discharge atmospheric pressure ionization source in helium. A resolution of at least 519 at $m / z 311$ is obtained, which is limited by the experimental parameters available in our experiment. The mass resolution is computer modeled and it is shown that as the mass increases, the experimental constraints become less important, and the resolution will increase. It is predicted that using the correct experimental conditions and with the addition of an ion reflector that resolution of well over 1000 should be obtained.
\end{abstract}

\section{INTRODUCTION}

Atmospheric pressure ionization (API) methods have provided a sensitive and rapid means of detecting trace amounts of organic species as well as a means of producing soft ionization for detection in combination with mass spectrometry. ${ }^{1-4}$ These advantages of API are due to the atmospheric pressure ionization sources, i.e., the $\mathrm{Ni} \beta$ source or corona discharge, which utilize kiloelectron volt electrons to ionize components in the air which then transfer a proton or charge, through a series of ion $/$ molecule reactions, to the analyte. In effect, this is atmospheric pressure chemical ionization and, because of the large number of collisions at atmospheric pressure, is highly efficient for molecules with high proton or electron affinities. In addition, because of the proton transfer mechanism and the large number of collisions, which carry away excess energy and minimize fragmentation, the molecular ion or the protonated molecule is observed for easy identification.

Atmospheric pressure ionization methods have recently received a great deal of attention since they are particularly well suited for analysis of organic compounds via liquid chromatography or liquid spray injection methods. ${ }^{5-7}$ In conventional HPLC/MS large amounts of liquid are sprayed into a vacuum system, which must pump away the liquid to produce a pressure that is sufficiently low $\left(10^{-5}\right.$ Torr) for the mass spectrometer to operate. However, in APIMS the liquid eluant is sprayed directly into a heated atmospheric pressure chamber, where the analyte molecules are ionized by a corona or $\mathrm{Ni} \beta$ source while the solvent is swept out of the chamber into an exhaust. Thus, the API method solves the pumping problem inherent in

1) Current address: Department of Electrical and Computer Engineering, SEMATECH Center, University of Arizona, Tucson, AZ 85721.

${ }^{b)}$ Current address: Cetac Tech., Omaha, NE 68131. other on-line liquid injection techniques. More recently, the introduction of ion evaporation methods, most notably electrospray, which can ionize large species such as peptides and proteins, have spurred the use of atmospheric pressure sources since the ion evaporation process must proceed under atmospheric pressure conditions. ${ }^{5,8}$

In this work, we have developed and modeled a time-of flight mass spectrometer which can be interfaced to an atmospheric pressure ionization source. This device utilizes a beam sweep method to generate a pulsed ion beam from a slit near the ion source for analysis by the TOF. In the past, API methodology has generally been interfaced to scanning mass spectrometers such as quadrupoles or magnetic sectors. However, TOF devices have several distinct advantages which include the ability to measure a full mass spectrum on every injection pulse and the ability to measure high mass with high transmission. In addition, TOF devices arc relatively simple and inexpensive and can achieve a resolution of 1000 or greater in combination with jet introduction ${ }^{9}$ or reflector techniques. ${ }^{10,11}$

In the present study, a high current atmospheric glow discharge source in helium ${ }^{12}$ is used to ionize various molecules of relatively low volatility injected into the API source via liquid injection. The resulting organic ions are introduced into a TOF, where, using a beam modulation technique, pulsed ion packets are generated which can be rapidly mass analyzed in the TOF. The beam sweep-modulation technique is modeled and optimized for the various experimental parameters. A resolution of at least 519 at $\mathrm{m} / z 311$ is obtained which is limited mainly by the experimental parameters in our present setup. It is shown that as the mass increases, these experimental constraints become less important, and the resolution will continue to increase, limited mainly by the sweep speed across the beam modulation slit. The capabilities and limitations of this novel TOF design are discussed. 


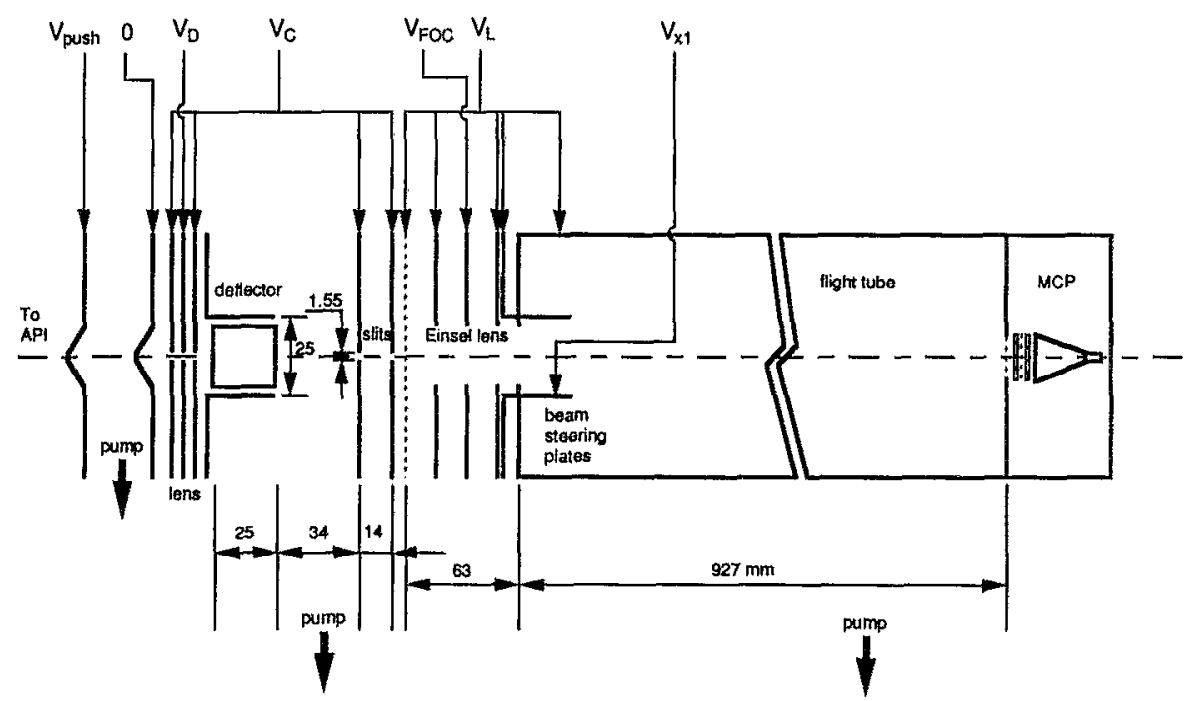

FIG. 1. The experimental setup where $V_{\text {push }}=130 \mathrm{~V}, \quad V_{D}=-200 \mathrm{~V}$, $V_{c}=-150 \quad \mathrm{~V}, \quad V_{\mathrm{L}}=-1000 \quad \mathrm{~V}$, $V_{\mathrm{FOC}}=-1400 \mathrm{~V}$ and $V_{X}=-960 \mathrm{~V}$. See Figs. 2 and 3 for the details of the API source and the deflector, respectively.

\section{EXPERIMENT}

The experimental setup is shown in Figs. 1 and 2. It consists of a differentially pumped API-TOF mass spectrometer (Fig. 1) (R. M. Jordan Co.) and a liquid injection sample source (Fig. 2). The liquid injection source delivers the sample dissolved in a solvent through a heated pneumatic nebulizer assembly to the vaporization chamber where the sample is vaporized and the solvent removed. The sample then passes through a duct into a separate second chamber and is ionized via a glow discharge source in 1-atm He. The resulting ions are injected through a pair of differentially pumped skimmers, which sample the onaxis component of the ion beam. The ions are transported in a jet flow of He carrier which narrows the translational energy spread of the injected ions. The ion beam passes through several focusing slits and is then swept across a collimating slit using a rectangular sweep motion via fourbeam deflection plates. This beam sweep triggers the start pulse for the TOF mass analysis and also determines the mass resolution of the instrument. The slit width and the speed with which the ion beam is swept across the slit

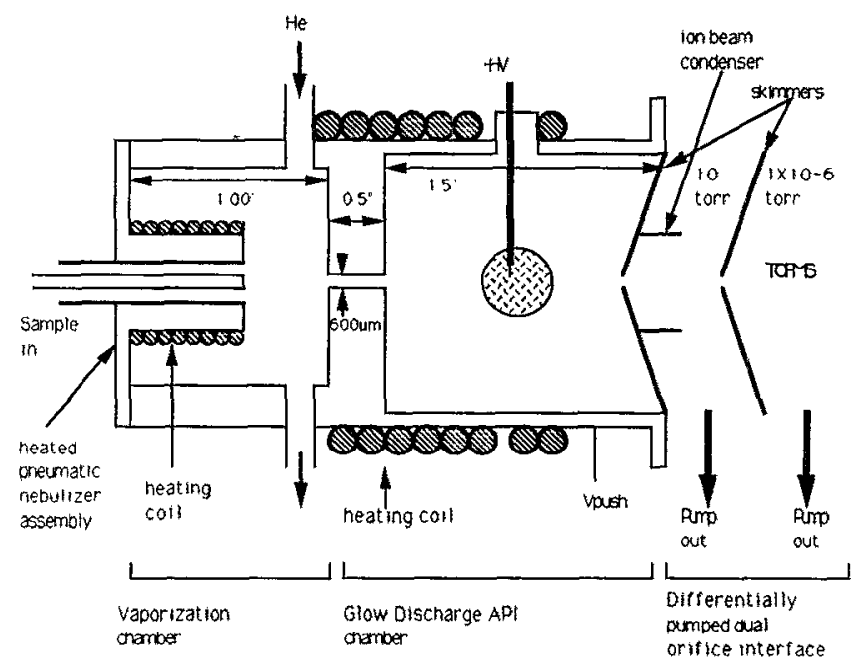

FIG. 2. Atmospheric pressure glow discharge source. determine both the throughput and the size of the ion packet introduced into the flight tube. The ion packet enters a field-free drift region 1-m long which is pumped to a pressure of $3 \times 10^{-7}$ Torr using a 4 -in. diffusion pump. The ions are detected at a triple-layer microchannel plate detector.

\section{A. Sample injection system}

In order to inject a variety of samples, including samples of relatively low volatility, into the TOF system, a liquid chromatography/atmospheric pressure ionization interface (LC/API) using a relatively high current glow in 1-atm He was developed (see Fig. 2). The system consists of a heated pneumatic nebulizer, a vaporization chamber, a glow discharge ionization chamber, and a differentially pumped dual orifice interface. The details of the system are as described below:

\section{LC}

An LC syringe pump (Varian Model 8500 or an ISCO Model $\mu$ LC-500 micropump) was used to deliver the eluant through the pneumatic nebulizer to the vaporization chamber. The samples were directly introduced into the vaporization chamber without the use of LC columns. The liquid flow rates were typically varied from $10 \mu \mathrm{L} / \mathrm{min}$ up to $150 \mu \mathrm{L} / \mathrm{min}$. The solvent was either 50:50 methanol/ water or pure methanol which was degassed by an ultrasonic bath before use.

\section{Nebulization}

The eluant was introduced to the vaporization chamber using a $50-\mu \mathrm{m}$ fused silica capillary tubing connected to a coaxial 1/16-in. stainless-steel tube with a 0.02 -in, i.d. The liquid was converted into fine droplets using a high velocity jet of helium (100 psi). The generated mist was then swept through a 1/8-in. heated stainless-steel tube with another helium gas stream $(100 \mathrm{~mL} / \mathrm{min})$ into the vaporizer. This helium fiow prevents the sample from depositing on the surface of the heated tube and conducts 
heat to vaporize the solvent. A 1/16-in. thermocoax heater was used to heat the $1 / 8$-in. SS tubing. The heated section is a 3/4-in. long, 1/2-in.-diam cylindrical brass block tightly wound with $1 / 16$-in. thermocoax cable heater through which the $1 / 8$-in. tube is inserted. This assembly is directly inserted and $o$-ring sealed to the back end of the vaporization chamber.

\section{Vaporization}

The vaporization chamber consists of a 1.0 -in. by $1.0-$ in. (diameter) cylindrical brass chamber and a heated brass block with a 0.5 -in. $\times 0.03$-in. (diameter) hole connected to the ionization chamber. A cross flow of dry gas (helium) towards the spray jet was employed to further remove the solvent vapor. The vaporized samples were transferred through the heated narrow channel into the ionizer. The flow of the dry gas was controlled between 50 and $200 \mathrm{~mL} / \mathrm{min}$ in order to optimize the signal. If a high liquid flow was present, then a correspondingly high dry gas flow was used in order to obtain a stable glow discharge.

\section{Ionization}

The ionization chamber is directly attached to the skimmer and consists of 1.0 -in. $\times 0.8$-in. (diameter) brass cell with two 1/4-in. Cajon ports with quartz windows for observation and one 1/8-in. Cajon port for the discharge electrode. The glow discharge is formed with the use of a 0.04-in.-diam tungsten rod which is ground to a sharp tip. The rod is covered with glass insulation and only the tip is exposed to prevent an unstable discharge from occurring on the side of the rod. The vaporized sample is ionized via ion-molecule reactions induced by the $\mathrm{He}$ glow discharge as described in previous work. ${ }^{12}$

\section{Atmospheric pressure interface}

A dual-orifice differentially pumped interface was developed to sample the ions from the atmospheric pressure ionization chamber. The first sampling orifice is $350 \mu \mathrm{m}$ and the second orifice inlet to the mass spectrometer is $\mathbf{5 2 0}$ $\mu \mathrm{m}$. The region between the two orifices is pumped by a $650-\mathrm{L} / \mathrm{min}$ mechanical pump to a pressure of $\sim 2$ Torr. The pressure in the mass spectrometer acceleration region under these conditions is $\sim 3 \times 10^{-6}$ Torr. An ion beam condenser with voltage $V_{\text {push }}$ is placed between the orifices to enhance the transmission efficiency of the ions into the mass spectrometer. $V_{\text {push }}$ is a voltage placed on the entire API source so that the voltage differential is between the two skimmers. The ions in the ionization region are extracted mainly by the rapid pumping of the He buffer by the interface region.

\section{Operation}

The temperatures of the pneumatic nebulizer and vaporization chamber can be controlled independently using a thermocouple and temperature controller. The typical working temperature was $190-230^{\circ} \mathrm{C}$. However, the actual temperature of the sample is expected to be much lower

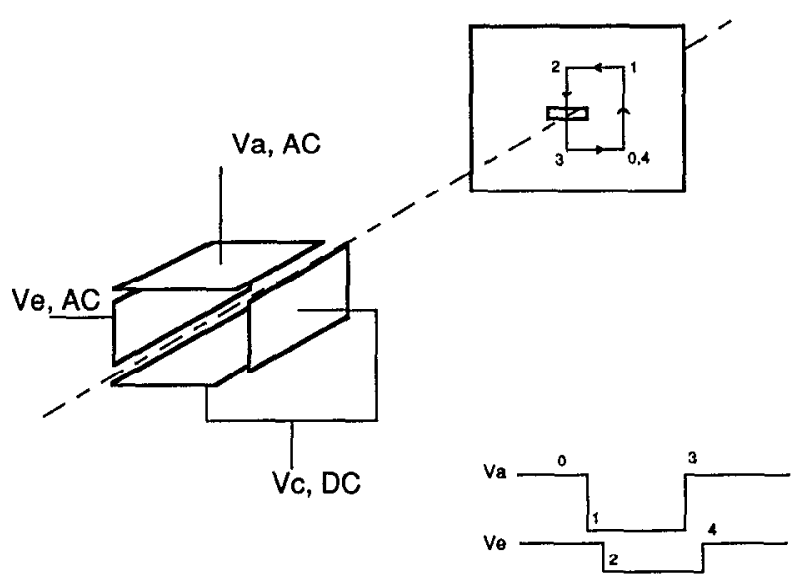

(a)

(b)

FIG. 3. (a) Ion pulse generation scheme using a sweep slit arrangement; (b) The variation of $V_{a}$ and $V_{e}$ for sweeping the ion beam across a slit.

than the body temperature of the vaporization chamber or pneumatic nebulizer since the heat is carried away by the vaporized solvent and carrier gas.

In order to initiate the glow discharge, -1 to $-2 \mathrm{kV}$ was applied to the electrode. When the glow was obtained, the value of the actual operating voltage was dropped as much as $100-500 \mathrm{~V}$ from the initial breakdown voltage and a current of $0.2-1 \mathrm{~mA}$ was maintained. The current could be continuously increased up to $10 \mathrm{~mA}$ when required. The resulting glow appeared as a white and blue glow extending from the tip of the electrode. The most stable glow was maintained in $1 \mathrm{~atm}$ of He. The gas flow of the nebulizer and the dry gas must be optimized together in order to achieve the best signal, especially when high liquid flow rates were used. Two fine control flow meters were used for this purpose.

\section{B. Ion packet generation}

A key feature of the API/TOF design is the ion packet generation method. A means of generating a pulse from a continuous ion beam source, as in API, is required in a TOF device in order to provide a start point $(t=0)$ for each spectrum. In the present experimental arrangement the ion beam enters on-axis with the flight tube. A set of ion deflector plates were used in order to generate the ion packet by sweeping the dc ion beam across a slit as shown in Fig. 3(a). Four metal plates form a rectangular box around the ion beam. The plates are 1-in. long in the beam direction and spaced $\sim 1$ in. apart. The sweep mechanism operates such that $V_{c}$ is a constant dc voltage which is applied to the lower and right side plates. The typical value for $V_{c}$ is $-150 \mathrm{~V}$. As shown in Fig. $3(\mathrm{~b})$, at $t=0$, both $V_{a}$ and $V_{e}$ are at ground potential and the result is that the ion beam is deflected to the lower right corner of the slit surface designated as position " 0 ". At this point the ion beam is blocked and cannot enter the flight tube. In the next step $V_{a}$ changes from $0 \mathrm{~V}$ to a negative voltage $(\sim-300 \mathrm{~V})$, and the ion beam moves from position " 0 " to position "1" in Fig. 3(a). After an adjustable delay of 


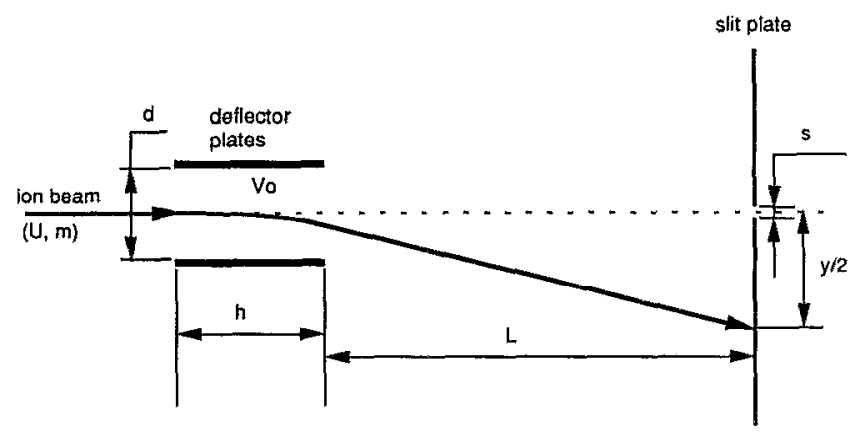

FIG. 4. One-dimensional deflector model, where $V_{0}$ is the potential difference between two plates.

100-600 ns, $V_{e}$ is raised to a voltage near $V_{c}$ and the ion beam moves from position " 1 " to " 2 ". Position " 2 " is now such that the ion beam is directly above the slit, but the ion beam is still blocked. $V_{a}$ is then switched to ground potential again after an adjustable duration (0-25 $\mu \mathrm{s})$ and the ion beam sweeps across the slit from position "2" to position " 3 ". The ion packet that reaches the detector is generated as the ion beam passes through the slit. The transit time from position "2" to position " 3 " is equal to the rise time of $V_{a}$ which was measured to be $100 \mathrm{~ns}$. After another adjustable delay time (100-600 ns), $V_{e}$ is dropped to low potential or ground and the ion beam moves to position "4" which corresponds to the initial position " 0 ". The next sweep cycle, which has the identical loop, will start whenever $V_{a}$ is triggered to high negative voltage again. The overlapped time duration during which both $V_{a}$ and $V_{e}$ are high should be sufficiently long so that the heaviest mass under study can pass through the deffection area or else the resolution of the heavier masses will be degraded. The time spacing between the beginning of the sweep cycles should also be sufficiently long so that the heaviest mass under study can pass through the slit and be detected without overlapping with the next spectrum. Our simulations show that for $\mathrm{m} / z 5000$ with the current setup, $7.8 \mu \mathrm{s}$ are required in the sweep overlapped time duration for this ion to pass through the deflector area and $160 \mu$ s are needed in the time spacing between the experimental cycles for the ions to reach the detector during the cycle.

If we assume no initial deflection field such that the ion beam will travel along the axis parallel between the deflection plates (i.e., no $\perp$ momentum components) in a onedimensional deflector, one can derive the distance swept out in the loop between position "2" and " 3 " on the slit plane as

$$
y=\left(\frac{1}{2}\right)\left(\frac{h}{d}\right)\left(\frac{V_{0}}{U}\right)(h+2 L),
$$

where $h$ and $d$ are the length and the spacing of the deflector plates, respectively, $L$ is the distance from the end of the deflector to the slit plates, $V_{0}$ is the potential difference on the deflector plates (which is assumed to be constant), and $U$ is the kinetic energy of the ion as shown in Fig. 4. A key point here is that the loop size is independent of the mass/charge ratio if we assume the above. The result is
TABLE I. Computer simulation: $m / z$ independence of the ion packet width, $\Delta t_{\text {packet* }}\left(V_{c}=V_{e}=-150 \mathrm{~V}, V_{a}=2 V_{c}, V_{\text {push }}=130 \mathrm{~V}, t_{\text {rise }}\right.$ $=100 \mathrm{~ns}$ ), where $t_{\text {def }}$ is the time spent by the ion inside the deflector area in $\mu \mathrm{s}, s / y(\%)$ is the ratio of the slit width to the displacement of the ion on the slit plane in percentage [position "2" to " 3 " in Fig. 3(a)], $\Delta t_{\text {packet }}$ is the ion packet width in ns generated from the slit.

\begin{tabular}{lccc}
\hline \hline $\begin{array}{l}\mathrm{m} / \mathrm{z} \\
(\operatorname{arnu})\end{array}$ & $\begin{array}{c}t_{\text {def }} \\
(\mu \mathrm{s})\end{array}$ & $\begin{array}{c}s / y \\
(\%)\end{array}$ & $\begin{array}{c}\Delta t_{\text {packet }} \\
(\mathrm{ns})\end{array}$ \\
\hline 10 & 0.347 & 6.5 & 6.5 \\
100 & 1.097 & 6.5 & 6.5 \\
500 & 2.453 & 6.5 & 6.5 \\
1000 & 3.470 & 6.5 & 6.5 \\
5000 & 7.758 & 6.5 & 6.5 \\
10000 & 10.972 & 6.5 & 6.5 \\
\hline
\end{tabular}

that although heavier masses experience a smaller acceleration than the lighter masses, heavier masses spend a longer time inside the deflector region. Thus, the mass effect is cancelled out and the deflected distance $y$ is the same.

The theoretical ion packet time width is determined as

$$
\Delta t_{\text {packet }}=t_{\text {rise }}(s / y) \text {, }
$$

where $t_{\text {rise }}$ is the rise time of $V_{a}, s$ is the size of the slit, and $y$ is the ion beam displacement between position " 2 " and "3" in Fig. 3(a). $\Delta t_{\text {packet }}$ is estimated to be 7 ns for our experiment. The size of the ion packet in our experiment may be broadened by several factors in the field-free flight region, most important of which is the thermal energy of the ions. These factors will be discussed in the section on mass resolution.

Alternatively, the ion packet could simply be swept back and forth across the slit rather than in the present configuration where the ion packet is only swept across the slit in one direction. However, the former configuration presents two distinct disadvantages. If the ion packet is swept across the slit in both directions, this would require using the leading edge of the pulse [Fig. 3(b)] to sweep in one direction and the trailing edge to sweep in the opposite

TABLE II. Computer simulation: Rise time $t_{\text {rise }}$ dependence of the ion packet width, $\Delta t_{\text {packet. }}\left(V_{c}=V_{e}=-150 \mathrm{~V}, V_{a}=2 V_{c t} V_{\text {push }}\right.$ $=130 \mathrm{~V}, m / z=200 \mathrm{amu})$, where $t_{\mathrm{def}}(\mu \mathrm{s})$ is the time spent by the ion inside the deflector area in $\mu \mathrm{s}, s / y(\%)$ is the ratio of the slit width and the displacement of the ion on the slit plane in percentage [position "2" to " 3 " in Fig. 3(a)], $\Delta t_{\text {packet }}$ (ns) is the ion packet width in ns generated from the slit.

\begin{tabular}{lccc}
\hline $\begin{array}{l}t_{\text {rise }} \\
\text { (ns) }\end{array}$ & $\begin{array}{c}t_{\text {def }} \\
(\mu \mathrm{s})\end{array}$ & $\begin{array}{c}s / y \\
(\%)\end{array}$ & $\begin{array}{c}\Delta t_{\text {packer }} \\
(\mathbf{n s})\end{array}$ \\
\hline 10 & 1.552 & 6.5 & 0.7 \\
20 & 1.552 & 6.5 & 1.3 \\
40 & 1.552 & 6.5 & 2.6 \\
60 & 1.552 & 6.5 & 3.9 \\
80 & 1.552 & 6.5 & 5.2 \\
100 & 1.552 & 6.5 & 6.5 \\
120 & 1.552 & 6.5 & 7.8 \\
140 & 1.552 & 6.5 & 9.1 \\
160 & 1.552 & 6.5 & 10.4 \\
180 & 1.552 & 6.5 & 11.7 \\
200 & 1.552 & 6.5 & 13.0 \\
\hline \hline
\end{tabular}


TABLE III. Computer simulation: $V_{c}, V_{e}$ and $V_{a}$ dependence of the ion packet width, $\Delta t_{\text {packet. }}\left(V_{c}=V_{e}, V_{a}=2 V_{c}, V_{\text {push }}=130 \mathrm{~V}, t_{\text {rise }}=100 \mathrm{~ns}\right.$, $m / z=200 \mathrm{amu})$, where $t_{\text {def }}$ is the time spent by the ion inside the defiector area in $\mu s, s / y(\%)$ is the ratio of the slit width and the displacement of the ion on the slit plane in percentage [position " 2 " to " 3 " in Fig. $3(\mathrm{a})$ ], $\Delta t_{\text {packet }}$ is the ion packet width in ns generated from the slit.

\begin{tabular}{llcc}
\hline$V_{c}$ & $\begin{array}{c}t_{\text {def }} \\
(\boldsymbol{V})\end{array}$ & $\begin{array}{c}s / y \\
(\mu \mathrm{s})\end{array}$ & $\begin{array}{c}\Delta t_{\text {packet }} \\
(\mathrm{ns})\end{array}$ \\
\hline-40 & 1.991 & 14.8 & 14.8 \\
-60 & 1.884 & 11.0 & 11.0 \\
-80 & 1.792 & 9.1 & 9.1 \\
-100 & 1.712 & 8.0 & 8.0 \\
-120 & 1.642 & 7.2 & 7.2 \\
-140 & 1.580 & 6.7 & 6.7 \\
-160 & 1.525 & 6.3 & 6.3 \\
-180 & 1.475 & 6.0 & 6.0 \\
-200 & 1.429 & 5.7 & 5.7 \\
\hline \hline
\end{tabular}

direction. The trailing edge and leading edge of the pulse usually have different rise times. The rise time will affect the resolution with the result that there will be two different sets of mass spectra with different resolutions. In addition, if the delay between the two spectra generated by the leading and trailing pulses, i.e., the pulse duration, is not sufficient, then the two spectra will overlap so that the heavy masses of one spectrum will be mixed with the lighter masses of the second spectrum. A duration of at least $160 \mu \mathrm{s}$ is required for $m / z 5000$, not to overlap with the second spectrum generated in the sweep mode. However, the present sweep circuit can only provide a maximum duration of $10 \mu \mathrm{s}$ at $1 \mathrm{kHz}$ repetition rate due to power limitations, so that the spectra will overlap. It should be noted that in the rectangular sweep configuration used in this work, it is the trailing edge of the voltage pulse which pulls the ion packet across the slit.

A computer simulation of the motion of the ion packet induced by the sweep circuit under various conditions was performed based upon the equations described above. This simulation was performed on a Compuadd 286 PC/AT. The results of the simulation for various parameters is given in Tables I-IV below. Tables I-IV show the effect of $\mathrm{m} / z, t_{\text {rise }}$ (pulse rise time), pulse voltage height, and push voltage $\left(V_{\text {push }}\right)$ on the packet width produced when the ion

TABLE IV. Computer simulation: $V_{\text {push }}$ dependence of the ion packet width, $\Delta t_{\text {packet. }}\left(V_{c}=V_{e}=-150 \mathrm{~V}, V_{a}=2 V_{c}, t_{\text {rise }}=100 \mathrm{~ns}, m / z\right.$ $=200 \mathrm{amu}$ ), where $t_{\text {def }}$ is the time spent by the ion inside the deflector area in $\mu \mathrm{s}, s / y(\%)$ is the ratio of the slit width and the displacement of the ion on the slit plane in percentage [position "2" to "3" in Fig. 3(a)], $\Delta t_{\text {pachet }}$ is the ion packet width in ns generated from the slit.

\begin{tabular}{lccc}
\hline $\begin{array}{l}V_{\text {push }} \\
(\mathrm{V})\end{array}$ & $\begin{array}{c}t_{\text {def }} \\
(\mu \mathrm{s})\end{array}$ & $\begin{array}{c}s / y \\
(\%)\end{array}$ & $\begin{array}{c}\Delta t_{\text {packet }} \\
(\mathrm{ns})\end{array}$ \\
\hline 10 & 2.053 & 3.7 & 3.7 \\
50 & 1.836 & 4.6 & 4.6 \\
100 & 1.642 & 5.6 & 5.6 \\
150 & 1.499 & 6.9 & 6.9 \\
200 & 1.388 & 8.1 & 8.1 \\
250 & 1.298 & 9.3 & 9.3 \\
300 & 1.224 & 10.4 & 10.4 \\
\hline \hline
\end{tabular}

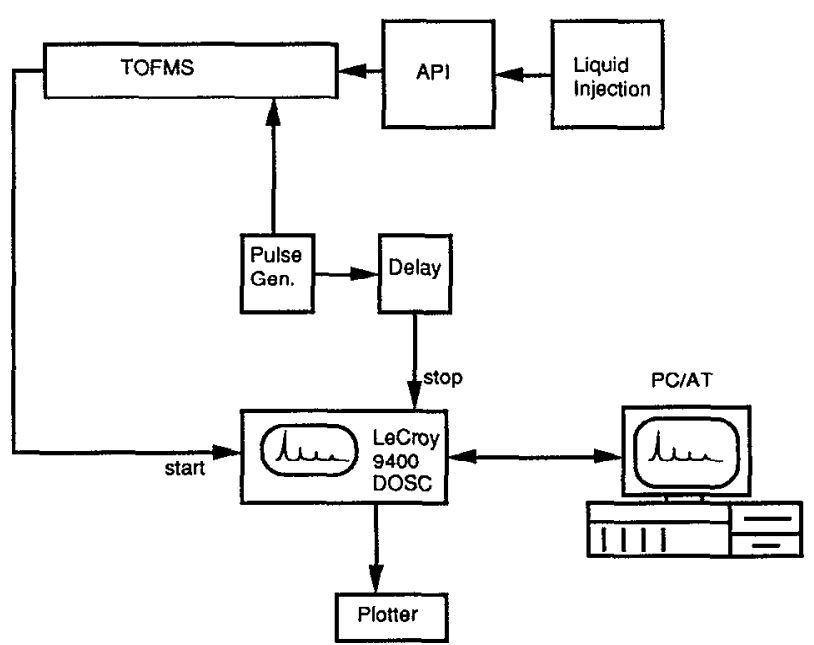

FIG. 5. Experimental arrangement for "single-hit" data collection mode.

beam is swept past the slit. Table I, for example, shows that under the conditions assumed in the last section that the ion packet width is not a function of the $m / z$. However, as expected, the rise time of the pulse, the sweep voltages, and the push voltage from the API source all play a critical role in determining the ion packet width. Table II shows the fact that as the rise time becomes longer, it takes longer to cross the slit, and thus the ion packet width increases. In Table III, one observes that as the deflection voltage increases, the size of the loop increases, and the ratio of $s / y$ decreases, so the ion packet width decreases. In Table IV as the push voltage, i.e., the bias voltage in the API chamber increases, the ion travels faster, so that the deflection is smaller and the ion packet width increases. This assumes that the travel time from point " 2 " to point " 3 " depends solely on the pulse, $V_{a}$, rise time. The results shown in the tables are given under the particular conditions of this simulation where $V_{c}=V_{e}$ and $V_{a}=2 V_{c}$. Under these conditions the ion beam exactly crosses the center of the slit and the distance from the slit to position "2" or " 3 " will be equal.

\section{TOF device}

Once the ions pass through the sweep slit region, they are collimated by several additional slits and focused using a set of Einsel lenses. A pair of beam steering plates are then used to steer the ions onto the channel plate. The flight tube uses a liner which is typically at a voltage of $-1000 \mathrm{~V}$ to pull the ions down the flight tube. The use of a liner in the flight tube allows the beam deflection setup to operate at relatively low voltage. The detector is a triplelayer microchannel plate $(18 \mathrm{~mm}$ diameter) with a gain estimated from previous work of $5 \times 10^{7}$ to $5 \times 10^{8} .{ }^{13}$ The face of the first plate is operated at $-3.5 \mathrm{kV}$ to $-5.0 \mathrm{kV}$ instead of the usual $-2200 \mathrm{~V}$ in order to achieve additional postacceleration and to increase the sensitivity for detection of ions. The flight tube is pumped by a Varian VHS 4 diffusion pump while the main chamber is pumped by a Varian VHS 6 diffusion pump. A restriction of 1-in. 
tubing is placed between the main chamber and flight tube so that the main chamber is typically run at a pressure of $3 \times 10^{-6}$ Torr while the flight tube is at $3 \times 10^{-7}$ Torr using $1 \mathrm{~atm}$ of $\mathrm{He}$ as the API buffer gas.

\section{TOF measurement}

A LeCroy 9400 digital oscilloscope (DOSC) was used for measuring the TOF of the ion packets. The DOSC has the capability to signal average up to $10^{6}$ different single wave forms in order to enhance the signal to noise ratio. In the case where there is a strong ion signal and the detection event rate is much greater than the experimental sweep rate, the so-called "multihit" experiment was performed where many ion pulses could be detected for each sweep pulse. In this experiment, a pulse generator externally triggered the DOSC and the sweep circuit at the same time. The ion signals from the MCP detector were sent to the input of the DOSC, and the time difference between various ion peaks and the trigger $(t=0)$ time provides the time-of-flight of each ion. The multihit experiment means that there may be many ion peaks observed within a TOF experimental cycle.

Alternatively, if the detection rate is less than the experimental rate, a "single-hit" experiment is used as shown in Fig. 5. In this case the DOSC was triggered to start by the detector signal instead of the external triggering pulse. The latter was delayed and sent to the input of the DOSC to stop the time measurement. The actual TOF will be the time of the delay minus the measured time. This is called a "single-hit" experiment since there was only one pulse generated by a pulse delay generator in each experimental cycle. The "single-hit" mode was used in cases where there was a low signal level so that wave forms were only added if there was a signal from the detector. If the "multihit" mode was used in cases where the event rate/experimental rate $=1 \%$, one could not observe a signal peak in the spectrum after 100 signal averaged spectra, since only 1 signal is averaged for 99 background waveforms.

\section{E. Data analysis}

The TOF spectra in the DOSC can be plotted out by dumping the screen to a 2-pen HP plotter. However, in order to store the data permanently for analysis, it was transferred to a computer. A RS232 interface bus was established between the DOSC and a PC/AT computer. A user written QuickBASIC program was used to control the transfer processes. The size for each data point was $8 \mathrm{bit}$ for most of our experiments, but a 16-bit data form was used more recently to improve the accuracy.

The raw data from DOSC was in ASCII form. It was converted into signed decimal form by a user-written program. The TOF spectra were calibrated against the background protonated water peak. From the calibration constant $k$ and the time difference, $\Delta T$, between the signal peaks and the protonated water peak, the mass/charge of those TOF peaks may be found as

$$
m / z=\left[\left(T_{0}+\Delta T\right) / k\right]^{2},
$$
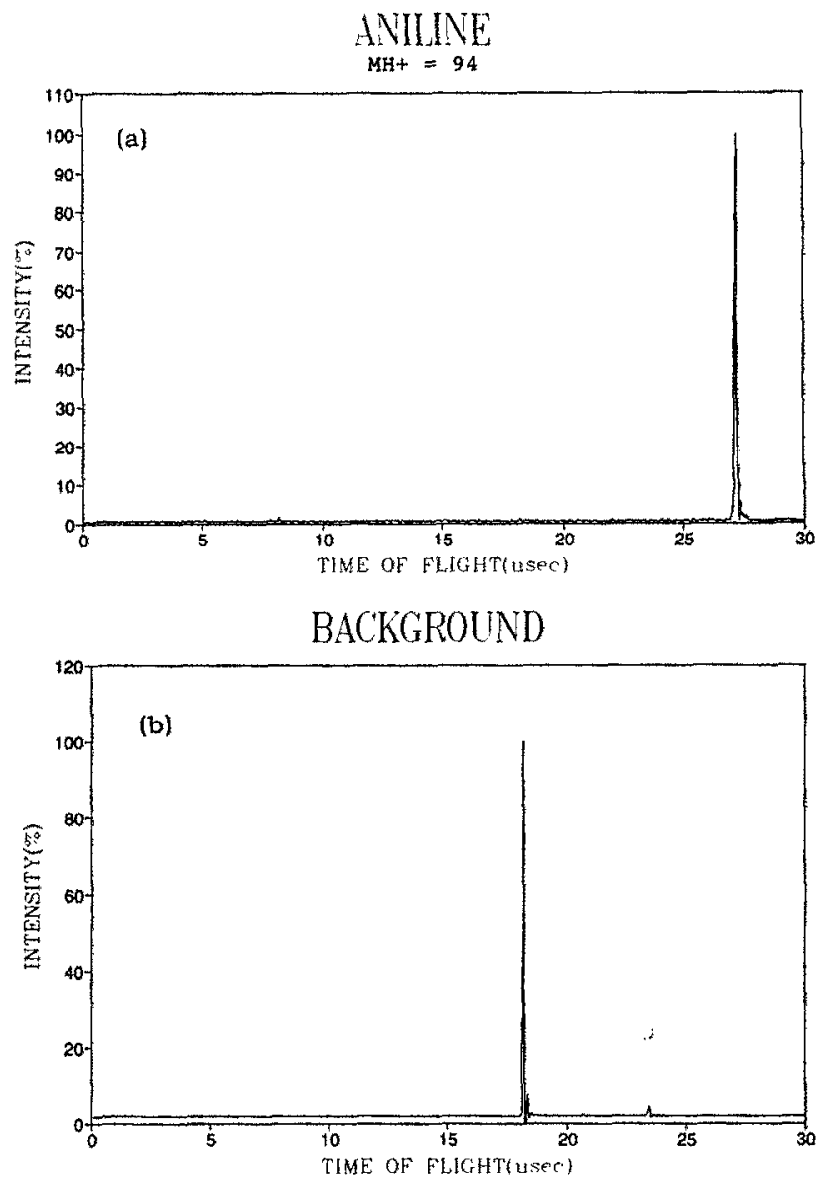

FIG. 6. Atmospheric pressure ionization/time-of-flight spectra of (a) aniline and (b) background methanol solvent.

where $T_{0}$ is the TOF of the protonated water.

\section{RESULTS AND DISCUSSION}

\section{A. Mass spectra}

The capabilities of the API/TOF device are demonstrated in the mass spectra of Figs. 6-8 and Table V. These spectra were obtained using high-pressure liquid injection from either methanol or methanol:water solvents into the heated nebulizer with ionization via the API glow discharge method in He. Figure $6(\mathrm{a})$ and $6(\mathrm{~b})$ shows the API/TOF mass spectra of aniline and solvent background injected into the API source from pure methanol solvent. These spectra were taken using a glow source discharge current of $\sim 0.4 \mathrm{~mA}$. The typical operating conditions for the sweep circuit in these experiments were $V_{c}=V_{e}$ $=-150 \mathrm{~V}, V_{a}=-300 \mathrm{~V}, V_{\text {push }}=175-190 \mathrm{~V}$ and $t_{\text {rise }}=100 \mathrm{~ns}$. The liner voltage was set at $-1000 \mathrm{~V}$, while the postacceleration triple MCP detector voltage was set at $-3900 \mathrm{~V}$ on the face of the first plate. The TOF spectra for aniline and the methanol solvent background shown in Figs. 6(a) and 6(b) were obtained using the "multihit" signal averaging method. The "multihit" method could be used in cases such as in 6(a) where the concentration was sufficiently high ( $1 \mathrm{mM}$ ) to obtain a strong signal. At low 
TABLE V. Liquid injection API mass spectra of sample molccules.

\begin{tabular}{lcc}
\hline \hline Sample & $m / z$ & Relative intensities \\
\hline Pyridine & $80 \mathrm{MH}^{+}$ & 100 \\
4-Iodoaniline & $220 \mathrm{MH}^{+}$ & 100 \\
& 94 & 20 \\
Thymidine & $243 \mathrm{MH}^{+}$ & 68 \\
& 127 & 100 \\
Methadone & $311 \mathrm{MH}^{+}$ & 100 \\
3-Bromoaniline & $174 \mathrm{MH}^{+}$ & 100 \\
& $172 \mathrm{MH}^{+}$ & 96 \\
Amitriptyline & $278 \mathrm{MH}^{+}$ & 100 \\
Aniline & $94 \mathrm{MH}^{+}$ & 100 \\
Background & $33\left(\mathrm{CH}_{3} \mathrm{OH}\right) \mathrm{H}^{+}$ & 100 \\
& $65\left(\mathrm{CH}_{3} \mathrm{OH}\right)_{2} \mathrm{H}^{+}$ & 4 \\
\hline \hline
\end{tabular}

concentrations, the "single-hit" mode invariably had to be used. The TOF spectra were plotted by a laser printer from the stored data files. These spectra were obtained by signal averaging over 2000 signal pulses and were taken in $\sim 10$ min limited by the rate at which the DOSC can accumulate the data using 32000 points per sweep. Note that the methanol reactant ions generated in the glow source disappear in the aniline mass spectrum where the proton transfer from methanol to aniline occurs almost completely. In addition, the API glow source is a soft ionization source, where only the protonated molecule appears in the mass spectrum. The full width at half maximum (FWHM) of the peaks in these spectra are $\sim 40 \mathrm{~ns}$ as measured from the computer digitized data.

Figure 7 shows the API/TOF mass spectrum of 3bromoaniline. This was injected from liquid methanol into the heated nebulization chamber at $175^{\circ} \mathrm{C}$. The conditions are otherwise similar to those of Figs. $6(\mathrm{a})$ and $6(\mathrm{~b})$. A protonated molecule parent ion is observed at $\mathrm{m} / z 172$ and 174 with no accompanying fragmentation. The two ion peaks correspond to the two isotopic peaks of $\mathrm{Br}$, i.e., ${ }^{79} \mathrm{Br}$ and ${ }^{81} \mathrm{Br}$. These two isotopes are present in a natural abundance of 50.5:49.5 and thus two almost equal peaks are observed in the TOF mass spectrum. The two peaks are

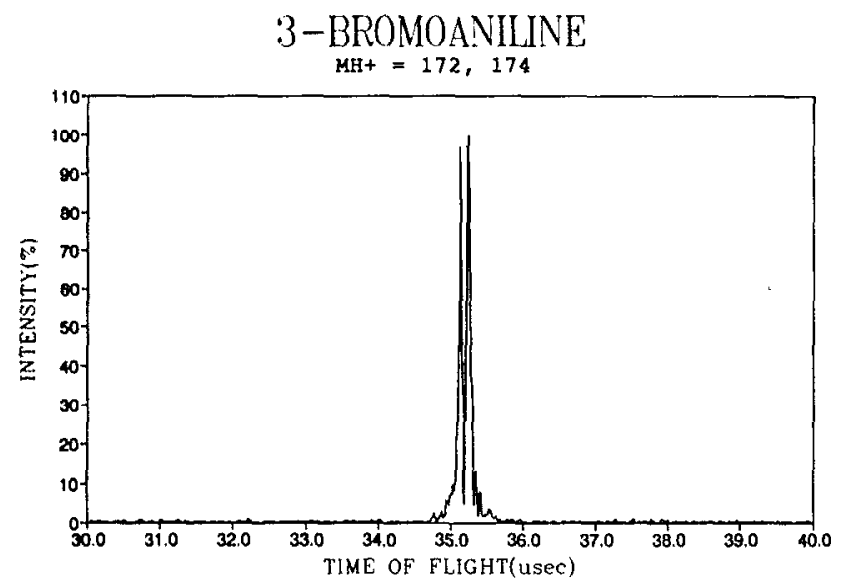

FIG. 7. Atmospheric pressure ionization/time-of-flight mass spectrum of 3-bromoaniline injected from liquid methanol solvent into the API source at $175^{\circ} \mathrm{C}$.

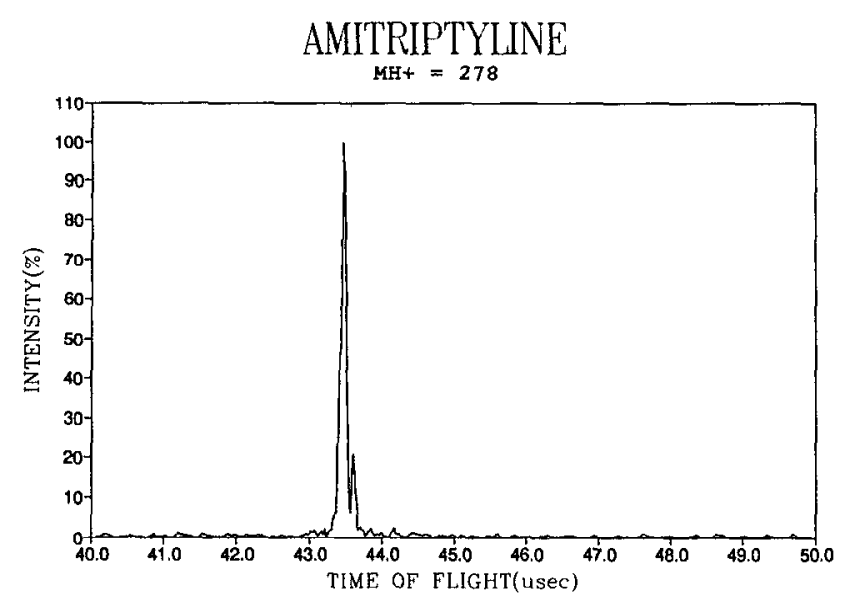

FIG. 8. Atmospheric pressure ionization/time-of-fight mass spectrum of amitriptyline injected from liquid methanol solvent into the API source at $225^{\circ} \mathrm{C}$.

separated almost to the base line, limited by the ${ }^{13} \mathrm{C}$ isotopic peak of the $3-{ }^{79}$ bromoaniline at $m / z$ 173. The FWHM peak width in this case is approximately $40-45 \mathrm{~ns}$. Thus a resolution of nearly 440 is obtained in this spectrum using the definition of Ref. 10. Figure 8 shows the API/TOF mass spectrum of the drug amitriptyline injected from liquid methanol at $225^{\circ} \mathrm{C}$. Only a protonated molecule peak, $\mathrm{MH}^{+}$, is observed at $m / z 279$ with no accompanying fragmentation. The peak at $m / z 280$ is the ${ }^{13} \mathrm{C}$ isotope peak which is present at about $20 \%$ of the amitriptyline parent ion. The isotopic peak is clearly resolved and the FWHM of the ion peaks are around 40-50 ns FWHM. Thus a resolution of $\sim 435$ is obtained in this spectrum.

In Table $V$ are tabulated the results of the API/TOF mass spectra for several organic compounds injected into the API glow source using liquid injection. For example, the API/TOF mass spectrum of 4-iodoaniline in Table V was injected from liquid methanol solvent into the API source at $190^{\circ} \mathrm{C}$. The elevated temperature is required to efficiently nebulize and vaporize this material which has a melting point of $62{ }^{\circ} \mathrm{C}$. A protonated molecule $\mathrm{MH}^{+}$at $m / z 220$ and an ion peak at $m / z 94$ was observed. The $m / z$ 94 peak arises from loss of iodine with replacement by a hydrogen in the hot high-pressure region. Thus some decomposition may occur at elevated temperature in this process. In addition, the nucleic acid base thymidine was studied. The working temperature of the API chamber was $230^{\circ} \mathrm{C}$ since the melting point of thymidine is $188^{\circ} \mathrm{C}$. In this case a protonated molecule $\left(\mathrm{MH}^{+}\right)$is observed at $m / z 243$; however, a second peak at $m / z 127$ represents cleavage between the base and sugar moiety with transfer of hydrogen from the sugar. This fragmentation is typical for these sugar bases in CI/MS. ${ }^{14}$ The API/TOF mass spectrum of the drug methadone injected from methanol solvent into the API source at $230^{\circ} \mathrm{C}$ was studied. A strong $\mathrm{MH}^{+}$peak is observed with no accompanying fragmentation under the conditions of this experiment. The concentration injected in these experiments is typically $1-10 \mu \mathrm{g} /$ $\mathrm{ml}$ over a period of 3-5 min using the "single-hit" method 
TABLE VI. Mass resolution.

\begin{tabular}{lcc}
\hline \hline Limiting factors & Contribution to $\Delta t$ & Mass resolution \\
\hline Ion packet width & $7 \mathrm{~ns}$ & 2857 for $t=40 \mu \mathrm{s}$ \\
PDG width & $30 \mathrm{~ns}$ & 666 for $t=40 \mu \mathrm{s}$ \\
DOSC resolution & $10 \mathrm{~ns}$ & 2000 for $t=40 \mu \mathrm{s}$ \\
Thermal energy & $1 / 2(\mathrm{~m} / 2 z U)^{1 / 2} \Delta U / U$ & $2500(U=1000, \delta=, 1)$ \\
Detector angle & $(\mathrm{m} / 2 z U)^{1 / 2} \Delta L$ & $2500(L=1000, \Delta L=, 4)$ \\
Total contribution & & 426 (low limit) \\
\hline
\end{tabular}

of data collection so that the total sample these spectra represent is $\sim 1$ nmole. However, sample levels down to $\sim 10 \mathrm{pg}$ (100-femtomolar) could be typically detected in these experiments using the triple-layer microchannel plate detector arrangement.

\section{B. Mass resolution}

One of the key issues in evaluating a TOF device is the resolution. The mass resolution in the sweep-slit arrangement used in this work is determined by several factors which can be easily calculated. In particular, for an ion with mass $m$ and kinetic energy $U$ traveling through a field-free region which has length $L$, the time-of-flight $t$, of the ion satisfies the following relationship with its mass $m$, such that

$$
m=(2 z U)(t / L)^{2} .
$$

The mass resolution is defined as $R=m / \Delta m$, where $\Delta m$ is the mass difference of two mass peaks which can be separated. Assuming $U$ and $L$ are fixed, one can obtain the mass resolution $R$ in the terms of $t$ and $\Delta t$, where $\Delta t$ can be considered as the full width at half height of the signal peak in the TOF spectrum.

$$
R=m / \Delta m=t /(2 \Delta t)
$$

The various factors involved in determining $\Delta t$ are listed in Table VI. The contribution to $\Delta t$ from the ion packet width generated by the sweep-slit arrangement is 7 ns as discussed previously. Since many of our experiments, especially at low concentrations, involved data collection via the "single-hit" mode, a delay pulse from a pulse delay generator was used to stop the timing. The width of the delay pulse is measured at $30 \mathrm{~ns}$. The LeCroy 9400 DOSC has a resolution of only 10 ns. By directly adding these time limiting factors together, a worst case resolution $R$ for a given TOF in the "single-hit" mode can be obtained. For $t=40 \mathrm{~ns}(m / z=314, U=1000 \mathrm{~V}, L=1000 \mathrm{~mm}), R$ $=426$ can be calculated from Eq. (4). In the "multihit" mode the method of data collection should not be limited by the width of the delay generator and indeed on a single pulse a pulse width of 10-12 ns FWHM is observed. However, in practice a pulse width of $\sim 40$ ns is observed due to the triggering instabilities in this method of data collection signal averaged over many pulses.

The $\Delta t$ contribution from the thermal energy spread and the limited detection aperture size are dependent on the mass/charge. Due to the initial ion beam energy dis- tribution, some of the ions tend to move away from the beam axis which will cause a time difference in the arrival of the ions at the detector. If we rearrange Eq. (3), then

$$
t=L \sqrt{m / 2 z U}
$$

and

$$
d t=L \sqrt{m / 2 z}(-1 / 2) u^{-3 / 2} d u,
$$

which implies that

$$
\Delta t=(1 / 2) \sqrt{m / 2 z U} \Delta U / U \text {. }
$$

Thus from Eq. (4) the resolution $R$ from this contribution will be

$$
R=t / 2 \Delta t=U / \Delta U .
$$

$R$ will be 2500 if $U=1000 \mathrm{~V}$ and $\Delta U=0.4 \mathrm{eV}$.

The detector aperture size is $18 \mathrm{~mm}$ which can contribute to $\Delta t$ due to the different paths of the ions that result from the momentum spread and thus the different angles that ions follow. This can be derived directly from Eq. (5) where

$$
d t=d L \sqrt{m / 2 z U},
$$

which implies that

$$
\Delta t=\sqrt{m / 2 z U} \Delta L
$$

and

$$
R=t / 2 \Delta t=L / 2 \Delta L
$$

One obtains $R=2500$ for $L=1000 \mathrm{~mm}$ and $\Delta L=0.2$ $\mathrm{mm}$. Notice that the contribution from both energy and path is not significant for mass/charge under 1000 . They will become important in studies in the higher mass range.

It should be noted that alternatively the slit can be placed directly in front of the detector and the ion beam swept across the slit using various sweep modes known as the "gate" mode or "differential impulse sweep" mode. ${ }^{15-19}$ In these cases, a continuous ion beam is swept into the TOF device and the equations governing the resolution for these modes of operation are very different from our methodology where a pulsed ion beam is injected into the flight tube. In the modes where a continuous ion beam is injected into the TOF, the resolution depends critically on the distance between the plates and the slit. In the

TABLE VII. Experimental ion peak widths and mass resolution. ( $V_{c}$ $=V_{e}=-150 \mathrm{~V}, V_{a}=2 V_{c}, V_{\text {rise }}=100 \mathrm{~ns}$, temperature $=190-230^{\circ} \mathrm{C}$, Liquid injection rate $=10-50 \mu \mathrm{L} / \mathrm{min})$, where $\left(\mathrm{MH}^{+}\right)$is the protonated molecular ion in the mass spectra, TOF is the time-of-flight of $\mathrm{MH}^{+}$ion in the units of $\mu \mathrm{s}$, FWHM is the full width at the half maximum of the ion peak in units of ns, and $R_{\text {expt }}$ is the experimental mass resolution obtained from the above spectra.

\begin{tabular}{lrrcc}
\hline \hline Sample $\left(\mathrm{MH}^{+}\right)$ & $m / z$ & $\begin{array}{c}\text { TOF } \\
(\mu \mathrm{s})\end{array}$ & $\begin{array}{c}\text { FWHM } \\
\text { (ns) }\end{array}$ & $\boldsymbol{R}_{\text {expr }}$ \\
\hline Water & 19 & 9.84 & 40 & 123 \\
4-Iodoaniline & 220 & 34.77 & 50 & 347 \\
Thymidine & 243 & 35.44 & 40 & 443 \\
Amitriptyline & 277 & 39.31 & 40 & 491 \\
Methadone & 311 & 41.49 & 40 & 519 \\
\hline \hline
\end{tabular}


TARLE VIII. Computer simulation: FWHM of the ion peaks due to the ion energy variation. $m / z$ is the mass charge ratio in the units of amu, $\Delta U$ is the average ion energy variation in the units of eV, FWHM is the full width at the half maximum of the ion peak in units of ns.

\begin{tabular}{rccc}
\hline$m / z \backslash \Delta U$ & 0.05 & 0.10 & 0.50 \\
\hline 20 & 5 & 5 & 6 \\
200 & 5 & 6 & 23 \\
500 & 5 & 8 & 30 \\
1000 & 6 & 9 & 39 \\
5000 & 10 & 17 & 98 \\
10000 & 14 & 23 & 122 \\
\hline
\end{tabular}

present work, the resolution does not depend on this parameter, but, rather, is limited by other factors as illustrated in Table VI. The alternative methods first developed by Bakker ${ }^{15,16,19}$ could also be used in this work. The advantages of the present methodology allow the production of a pulsed ion beam for experiments where this might be required, e.g., photodissociation. In addition, in the Bakker methodology, a continuous ion beam traverses the flight tube and impinges on a slit near the detector. The ion sputtering on the slit piece may cause ejection of ions that may result in a continuous background on the TOF spectrum.

As discussed above, the delay pulse and DOSC resolution together contribute $\sim 40$ ns to the pulse width $(\Delta t)$ in the "single-hit" mode and the pulse width in the "multihit" mode is also typically limited to $\sim 40$ ns. These factors are mass independent. In the case of heavier mass, i.e., longer flight time, the mass-dependent factors that limit resolution become more important. We thus measured the resolution for several ion peaks ranging in size from water clusters, $m / z 19$, to the methadone protonated molecular peak, $\mathrm{MH}^{+}$, at $m / z 311$ as shown in Table VII. The conditions of the TOF mass spectra are slightly different from those obtained in Figs. 6-8 and thus the flight times are different. The operating conditions are given in the legend of Table VII. The FWHM peak width was determined from the digitized data obtained by the DOSC and stored in the computer. Since the time resolution does not change after transfer to the computer, the width measurement is the same as that directly read from the oscilloscope. The TOF of methadone, $\mathrm{MH}^{+}$, for example, was measured to be $41.49 \mu$ s under the conditions of the experiment while the peak width FWHM was 40 ns. From Eq. (4), a mass resolution $R$ of 519 is obtained. Notice that the lower resolution is measured for smaller ions due to the dominant contribution of the mass-independent factors that limit the resolution in the lower mass region. As the mass increases, the contribution of the mass-dependent factors to the resolution increases, and the overall resolution will increase. It should be noted that this resolution was obtained in a simple linear TOF and that the resolution could be easily improved to over 1000 with the use of a reflector device due to the longer flight path.

One of the major factors limiting the resolution of TOF devices is the energy spread of the ions resulting from the Boltzmann velocity distribution of neutrals. ${ }^{20}$ Al room temperature this can be estimated at $\sim 0.09 \mathrm{eV}$ and includes the fact that the ions initially produced may be moving in opposite directions. In the present source where ions are injected into vacuum in a jet of $\mathrm{He}$ from 1-atm back pressure, the ions are moving in the same direction towards the slit so that the energy spread is reduced by at least a half. In addition, the effect of the jet cooling will further reduce the energy spread. However, assuming a worst case scenario for the energy spread $(\Delta U)$ in this setup, a simulation was performed to study the effect of the ion energy variation on the ion peak width. In this simulation (see Table VIII) the ion energy spread was assumed to be a Gaussian distribution and the Monte Carlo method was used to generate the ion energy for each ion. The ion energy generated was randomized but satisfied the Gaussian distribution. The mean energy of the distribution was determined by experimental factors, i.e., $V_{c}, V_{\text {push }}, V_{L}$, etc., while the energy spread $\Delta U$ was the parameter introduced into the simulation. Of course, in the simulation only ions that pass through the slit system can contribute to the mass spectra. Initially the ion packet width was $5 \mathrm{~ns}$ in the exit of the slit under the conditions of this calculation. The time resolution in this simulation was $1 \mathrm{~ns}$. The peak width (FWHM) was obtained when the ion peak height reached 2000 counts in order to improve the statistical error. As shown in Table VIII the ion peak width increases as the mass or energy spread increases. The mass resolution decreases significantly as the energy spread increases, especially for the higher mass range. However, under the conditions of these experiments, i.e., mass range $200-500 \mathrm{u}$ and an energy spread $<0.05 \mathrm{eV}$, one would not expect the energy spread to play a significant role in determining the resolution.

\section{Duty cycle}

One of the great inherent advantages of TOF devices is the improvement in duty cycle over scanning mass spectrometers. However, the use of a sweep slit method severely degrades this capability. The actual time the ion packet passes the slit is only 6-10 ns per cycle. Assuming that at least $100-150 \mu \mathrm{s}$ is required to observe an entire mass spectrum and to sweep the entire cycle, then a maximum sweep repetition rate of $10 \mathrm{kHz}$ would be possible. This provides a duty cycle of $10^{-4}$. In practice, a rapid integrating recorder (ITR) would be required to take advantage of this high repetition rate, which cannot be accommodated by the DOSC used in this work. However, assuming an ITR is available, then this would compare favorably to a scanning mass spectrometer, i.e., quadrupole, magnetic sector, where only one mass is observed at a particular time so that for a mass range of $1000 \mathrm{u}$ a duty cycle of $10^{-3}$ is obtained. However, the resolution in our device is not limited by the sweep slit, but rather by other experimental factors. Thus, the slit size could be increased by a factor of 3-4 in which case the sweep slit TOF duty cycle would become very comparable to that of scanning devices. The duty cycle could be enhanced further by using an ion storage device to store part of the continuous ion beam during those phases of the sweep cycle where the ions 
are not being swept past the slit. Thus, the duty cycle of the API/TOF device could be made comparable to or better than that of scanning mass spectrometers. In practice, though, in quadrupole mass spectrometers, as an example, the ion transmission is limited by the small acceptance angle and aperture required. More important, in quadrupole devices the ion transmission tends to fall rather rapidly as a function of $m / z$. Thus, as the $m / z$ increases the ion transmission of the sweep slit TOF device can become increasingly better than that of a quadrupole despite the initial duty cycle considerations. This may become true, especially at masses over 500 where the transmission of many quadrupoles may become very low, i.e., less than $1 \%$. However, one may expect to study increasingly higher masses in a TOF without significant loss in transmission. It is in this situation where the API/TOF will become a powerful tool for analysis. In addition, the ultimate improvement of TOF comes in experiments where a pulsed ion source is required, such as photodissociation experiments. In this case ion packets are produced which can be photodissociated by pulsed laser sources. The resulting ion products can be readily detected by a TOF device, but cannot be easily detected within the sweep cycle of a scanning device.

\section{ACKNOWLEDGMENTS}

We gratefully acknowledge support of this work by the Army Research Office under Grant DAAL 03-88-K-0191 and by the National Center for Human Genome Research under Grant No. 1 P30 H600209. David M. Lubman is an Alfred P. Sloan Research Foundation Fellow.

${ }^{1}$ D. I. Carroll, I. Dzidic, E. C. Horning, and R. N. Stillwell, Appl. Spectrosc. Rev. 17, 337 (1981).

${ }^{2}$ M. W. Siegel and W. L. Fite, J. Phys. Chem. 80, 2871 (1976).

${ }^{3}$ H. Kambara, Y. Ogawa, Y. Mitsui, and I. Kanomata, Anal. Chem. 52, $1500(1980)$

${ }^{4}$ J. V. Iribarne, P. J. Dziedic, and B. A. Thomson, Int. J. Mass Spectrom. Ion Phys. 50, 331 (1983).

${ }^{5}$ E. C. Huang, T. Wachs, J. J. Conboy, and J. D. Henion, Anal. Chem. 62, 713A (1990).

${ }^{6} \mathrm{M}$. Sakairi and H. Kambara, Anal. Chem. 61, 1159 (1989).

${ }^{7}$ E. C. Horning, D. I. Carroll, I. Dzidic, K. D. Haegele, M. G. Horning, and R. N. Stillwell, J. Chromatogr. 99, 13 (1974).

${ }^{8}$ J. B. Fenn, M. Mann, C. K. Meng, S. F. Wong, and C. M. Whitehouse, Mass Spectrom. Rev, 9, 37 (1990).

${ }^{9}$ D. M. Lubman and R. M. Jordan, Rev. Sci. Instrum. 56, 373 (1985).

${ }^{10}$ B. A. Mamyrin, V. I. Karataev, D. V. Shmikk, and V. A. Zagulin, Sov. Phys. JETP 37, 45 (1973).

${ }^{11}$ U. Boesl, H. J. Neusser, R. Weinkauf, and E. W. Schlag, J. Phys. Chem. 86, 4857 (1982).

${ }^{12}$ I. Sofer, J. Zhu, H. S. Lee, W. Antos, and D. M. Lubman, Appl. Spec. 44, 1391 (1990).

${ }^{13}$ C. J. Amentrout, Rev. Sci. Instrum. 56, 1179 (1985).

${ }^{14}$ M. S. Wilson and J. A. McCloskey, J. Am. Chem. Soc. 97, 3436 (1975).

is J. M. B. Bakker, J. Phys. E 6, 785 (1973).

${ }^{16}$ J. M. B. Bakker, J. Plyss. E 7, 364 (1974).

${ }^{17}$ J. H. Futrell, T. D. Tiernan, F. P. Abrahamson, and C. D. Miller, Rev. Sci. Instrum. 39, 340 (1968).

${ }^{18}$ K. Schey, R. G. Cooks, R. Grix, and H. Wollnik, Int. J. Mass Spectrom. Ion Proc. 77, 49 (1987).

${ }^{19}$ G. E. Yefchak, G. A. Schultz, J. Allison, C. G. Enke, and J. F. Holland, J. Am. Soc. Mass Spectrom. 1, 440 (1990).

${ }^{20}$ W. C. Wiley and I. H. McLaren, Rev. Sci. Instrum. 26, 1150 (1955). 